\title{
Be STARS IN YOUNG LMC CLUSTERS *
}

\author{
H. KJELDSEN, D. BAADE \\ European Southern Observatory \\ Karl-Schwarzschild-Str. 2, D-85748 Garching bei München, Germany
}

\begin{abstract}
Slitless field $\mathrm{H} \alpha$ spectroscopy with a resolution of $0.2 \mathrm{~nm}$ has detected numerous Be stars in 3 young open star clusters in the LMC but only a few each in one Galactic and one LMC cluster. The line widths indicate rapid rotation as is typical of Galactic field Be stars. In NGC 2004 (LMC) their distribution seems inconsistent with the assumption of either very similar equatorial rotation velocities or random orientation of the rotation axes or both, which are believed to be valid for Galactic field Be stars.
\end{abstract}

\section{Introduction and Observations}

There is no widely accepted model which predicts either which rapidly rotating B stars (Bn stars) become Be stars or, if all Bn stars transform into Be stars, when this happens. In the Galaxy (Bright Star Catalog), an average $\sim 10 \%$ of the B-type stars show line emission and roughly twice as many between B1 and B3. The open star cluster NGC 330 in the SMC has become known for its unusually high content of Be stars (e.g., Grebel et al. 1992). Since NGC 330 possibly was the first Magellanic Cloud cluster which has been looked at in this regard, other clusters may be expected to show a similar excess of Be stars. In fact, in the LMC clusters NGC 2004 (Grebel et al. 1993) and 2100 (Bessell and Wood 1993) the same symptoms have been found since.

We have obtained slitless grism spectra with ESO's 3.5-m NTT at a dispersion of $0.2 \mathrm{~nm} / \mathrm{mm}$ and over a field of about $7 \times 7 \mathrm{arcmin}^{2}$. Each cluster was observed twice, with the grism being rotated through 90 degrees for the second exposure in order to reduce crowding problems. For the same reason the length of the spectra was limited to $7.2 \mathrm{~nm}$ (FWHM) with an interference filter roughly centered on $\mathrm{H} \alpha$. In each 15 minute exposure, stars down to $m_{V}$ between 20 and 21 were detected which at a distance of $55 \mathrm{kpc}$ (LMC) covers all B-type stars on the main sequence and beyond.

\section{Detections}

From a preliminary analysis, we have so far found $17 \mathrm{Be}$ stars in NGC 1818 (LMC), 43 in NGC 2004 (LMC), 30 in NGC 2100, 3 in NGC 2122, and 2 in NGC 3293 (Galaxy). Since only limited photometry and spectral classification is available from the literature, it is not presently possible to reliably estimate the fraction of B-type stars with emission lines. However, the following conclusions are justified:

* Based on observations obtained at the European Southern Observatory, La Silla, Chile 
1. The widths of the $\mathrm{H} \alpha$ emission lines detected by us rule out unresolved HII regions as their origin. They strongly support the assumption that these Be stars belong to only one population, which furthermore is very similar to the Galactic one, as is also suggested by their homogeneous photometric variability (Balona 1992).

2. Both high and low proportions of Be stars occur in low- (SMC, LMC) as well as solar-metallicity environments (for instance, $50 \%$ of all early-B type stars in $\chi$ Per are Be stars, Schild 1965).

3. Clusters rich in Be stars may be more common in the Magellanic Clouds.

4. The Be stars in the LMC clusters observed by us are spread over the full field of view and appear less concentrated to the nucleus than the other cluster members.

\section{NGC 2004}

Age determinations for this LMC aggregate cluster around $10 \mathrm{Myr}$ but also reach $40 \mathrm{Myr}$. The metallicity $[\mathrm{Fe} / \mathrm{H}]=-0.7 \pm 0.1$. Grebel et al. (1993) find $\sim 55$ photometric Be star candidates. This is consistent with our census of 43 Be stars. Bessell and Wood (1993) also report a large, but unspecified, proportion of Be stars. We estimate a mean fraction of $30 \%$ Be stars which may peak at $45 \%$ roughly around B3-B4. Our numbers refer to the full field and, because of contamination by non-cluster members, only mark a lower limit. Equivalent widths (uncorrected for underlying $\mathrm{H} \alpha$ absorption) range from 0.2 to $11.5 \mathrm{~nm}$ with the peak of the line emission reaching up to ten times the continuum level.

After coarse correction for instrumental broadening, the line widths (FWHM) were found to lie between 100 and $500 \mathrm{~km} \mathrm{~s}^{-1}$. A histogram (bin width $50 \mathrm{~km} \mathrm{~s}^{-1}$ ) shows a broad plateau between 225 and $475 \mathrm{~km} \mathrm{~s}^{-1}$ which appears irreconcilable with the assumption (found to be acceptable for Galactic field Be stars, Lucy 1974) of roughly identical equatorial velocities and random orientation of the rotation axes. One may wonder whether in a young cluster the latter assumption is well satisfied by single stars.

\section{References}

Balona L.A.: 1992, MNRAS 256, 425

Bessell M.S., Wood, P.R.: 1993, in New Aspects of Magellanic Cloud Research, B. Baschek, G. Klare, J. Lequeux (eds.), Springer, Lect. Notes in Phys. 416, 271

(Grebel E.K., Richtler T., de Boer K.S.: 1992, $A$ \& $A$ 254, L5

Grebel E.K., Richtler T., de Boer K.S.: 1993, in New Aspects of Magellanic Cloud Research,

B. Baschek, G. Klare, J. Lequeux (eds.), Springer, Lect. Notes in Phys. 416, 366

Lucy L.B.: $1974, A J \mathbf{7 9}, 745$

Schild R.E.: 1965, ApJ 142, 979

(The full results will be submitted to Astronomy \& Astrophysics when the analysis has been completed.) 